\title{
Traction System Scheduling to Minimize Harmonic Current Level at Substation by Genetic Algorithm
}

\author{
YE Zhongming, LO Edward, PONG M. H., YUEN K. H. \\ Dept of Electrical \& Electronics Engineering \\ The University of Hong Kong \\ Pokfulam Road, Hong Kong, PRC
}

\begin{abstract}
Harmonics of individual trains are closely related to its loading, speed and operation mode. The harmonic current at substations is the sum of the individual components from all the trains electrically connected to the substation. There will be cancellation of the harmonics if the harmonics are not of the same phase angles. It is possible to schedule the traction system so as to minimize the harmonic distortion, improve the power factor and reduce the harmonic currents at substations. In this study Genetic Algorithm (GA) is used to find out the optimal schedule of the system with minimum harmonic levels. The optimized solution can be integrated into automatic train operation (ATO) controller to control the departure, speed regulation of each train of the system. Mathematical description of the problem is first presented and the genetic algorithm is introduced. The optimal solution is given at the end of this paper. It is demonstrated that the scheduling of traction system is applicable to harmonic reduction and GA is fit for such kinds of optimization problems. Such method of harmonics reduction can bring about considerable saving in filtering equipment.
\end{abstract}

Keywords: Harmonics, Traction System, Genetic Algorithm

\section{INTRODUCTION}

Modern electrified traction drives obtain power through phase controlled thyristor converters. During motoring and regenerative braking, the converter units of the train generate great amount of harmonic current. The increasing of passengers of mass transit system requires more frequent services, that is, more trains will be put into service. This will exacerbate the power quality especially harmonics problem of the traction system. To accommodate the increasing demand of harmonic power, usually installation of power filters and power factor correction equipment is necessary. Although some traction companies defer the responsibility to the power supply companies, many of them also expect to solve these problems with their own efforts.

The harmonic current of phase controlled thyristor converters is closely related to the loading, speed and operation mode of the drive. Since the trains keep moving within the traction system, the system configuration of the electric topology of the power system changes. The harmonics voltage and current at substation are determined by the harmonics generated by the trains which are electrically connected to the substation. Harmonics of same frequency from different trains may cancel each other, since each train may generate different harmonics, both different in magnitude and in phase angle. Thus it is possible to schedule the traction system so as to let more harmonics cancel each other and minimize the harmonic distortion, improve the power factor and/or reduce the harmonic currents. Each of the starting time and speed profile can be dynamically optimized so as to achieve the goal. The optimization solution can be integrated into the automatic train operation (ATO) controller to control the departure, speed regulation of each train on the system.

Genetic Algorithm (GA) can be used as optimization tool to find the optimal solution of the problems at hand. Genetic Algorithm has many advantages over the traditional gradient based optimization methods, for instance, GA searches the sub-optimal solution from a population of points, not a single point; GA use objective function's value and information, not derivation or other auxiliary knowledge, thus does not require the continuity of the objective function.

In this study, a method based on harmonics cancellation is proposed to minimize average harmonic current distortion at substation. Genetic algorithm is adopted for the optimization. The optimized schedule of the traction system can be integrated to the existing traction control system.

\section{BASIC MODEL OF SYSTEM AND RECTIFIERS}

For a mass transit system, in order to increase the energy efficiency of the power supply system, regenerative braking is employed together with the rheostat braking. During regenerative braking, the power is either absorbed by nearby trains or returned to the power system through converters in the nearby substations. For a given train running from the current station to the distinction station, ignoring those temporary stops, we assume the speed profile of trains can be represented by several key parameters including the starting time, motoring time, coasting time, braking time and running time $\left[x_{S}, x_{A}, x_{C}, x_{D}, T\right]$ as illustrated in Fig. 1, where suffix $S$ denotes starting, $A$ denotes motoring, $C$ denotes coasting and $D$ denotes braking. For long distance run, such as inter-city railway system, the motoring and coasting operation modes may repeat more than one time to cover long distance. 
However, for an urban mass transportation system, distance between stations is not very long, usually only one motoring and coasting occur during an inter-station run.

The distance between every two successive stations is definite and known, and the time period $T$ is equal to the starting time for the next station minus the starting time of this station, that is,

$$
\begin{aligned}
& d=\frac{1}{2} a \cdot x_{A}^{2}+a \cdot x_{A} \cdot x_{C}+\frac{1}{2} a^{\prime} \cdot x_{D}^{2} \\
& T=x_{S(i)}-x_{S(i+1)}
\end{aligned}
$$

and the running time here should be a sum of motoring time, braking time, coasting time plus the parking time at stations. In the above, $a$ and $a^{\prime}$ stand for acceleration speed and deceleration speed respectively.

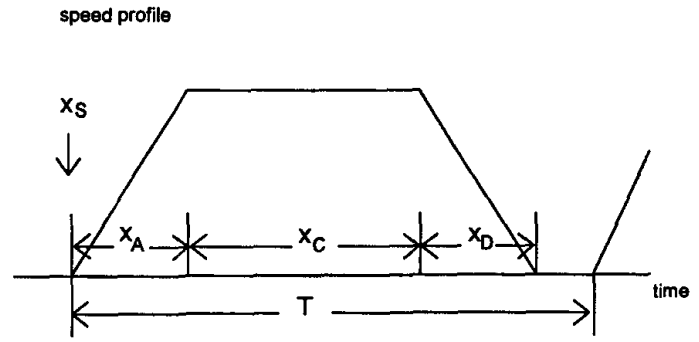

Fig. 1. The speed profile of trains.

If we further assume each train accelerates and brakes with the same rate, that is,

$$
\begin{aligned}
& \text { with the same rate } \\
& \qquad a=a^{\prime}
\end{aligned}
$$

hence $x_{A}=x_{D}$.

Then there will be only two sets of design unknowns $\left[x_{S}, x_{A}\right]$ for each train for a given station.

According to the motion equation, the armature current of dc motor can be given in the form,

$$
\begin{array}{ll}
I_{d}=\left(V_{d}-E\right) / R, & \text { for motoring, } \\
I_{d}=\left(V_{d}+E\right) / R, & \text { for braking. }
\end{array}
$$

where, the DC voltage imposed to the motor is the output of phase controlled circuit, if the firing angle is $a_{F}$, then $\mathrm{DC}$

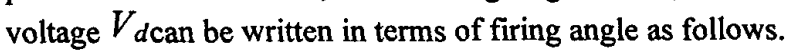

$$
V_{d}=V_{d 0} \cos a_{F}
$$

where $V_{d 0}$ is the maximum voltage.

The emfs for different operation modes are

$$
E(t)=E_{0}\left(t-x_{s}\right) / x_{A},
$$

$$
\begin{gathered}
\text { for motoring operation mode, } \\
E(t)=E_{0,} \\
\quad \text { for coasting operation mode, } \\
E(t)=E_{0}\left[1-\left(t-x_{S}-x_{A}-x_{C}\right)\right] / x_{D}, \\
\quad \text { for regenerative braking mode. }
\end{gathered}
$$

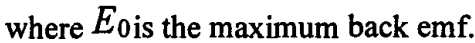

Then the current of the rectifier circuit can be given by the following Fourier series:

$$
i=\frac{2 \sqrt{3}}{\pi} I_{d} \sum_{h=1} \frac{1}{h} \sinh (\omega t+\beta)
$$

where $h$ is the order of harmonics, generally up to 19th harmonics is sufficient for traditional harmonics analysis of traction system, $p$ is the number of phases:

$$
h=p s \pm 1 ; s=0,1,2,, \ldots
$$

and

$$
\begin{aligned}
& \beta=a_{F} s=0,2,4, \ldots \\
& \beta=a_{F} \pm \pi / h s=1,3,5, \ldots
\end{aligned}
$$

\section{THE OPTIMIZATION PROBLEM}

The goal is to find the minimum average overall total harmonic distortion of current at substations over a period of time for a system with $\mathrm{M}$ trains and $\mathrm{N}$ stations by optimal scheduling of the departure time and speed profile of every train in the system.

$$
\text { Obj: } \min \sum_{t=T_{0}}^{T_{N}} O T H D I_{t}\left(x_{S}, x_{A}, x_{D}\right)
$$

or to minimize the overall harmonics current at substations for a period of time,

Obj: $\min \sum_{t=T_{0}}^{T_{N}} I_{t}\left(x_{S}, x_{A}, x_{D}\right)$.

$$
\begin{aligned}
& \begin{array}{l}
\text { subject to: } \\
X_{S 1} \leq x_{s i j} \leq X_{S 2} \\
\quad \text { for } \mathrm{i}=1,2, \ldots, \mathrm{M} \text {, and } \mathrm{j}=1,2,3, \ldots, \mathrm{N} . \\
X_{A 1} \leq x_{A i j} \leq X_{A 2} \\
\quad \text { for } \mathrm{i}=1,2, \ldots, \mathrm{M} \text {, and } \mathrm{j}=1,2,3, \ldots, \mathrm{N} . \\
X_{C 1} \leq x_{C i j} \leq X_{C 2} \\
\text { for } \mathrm{I}=1,2, \ldots, \mathrm{M}, \text { and } \mathrm{j}=1,2,3, \ldots, \mathrm{N} .
\end{array}
\end{aligned}
$$

The maximum and minimum values $\left(X_{S 1}, X_{S 2}\right)$, $\left(X_{A 1}, X_{A 2}\right),\left(X_{C 1}, X_{C 2}\right)$ of potential solutions can be obtained from the historic records of real situation from the system operation. We use statistic evaluations from field measurement of the traction system for this study. 
The harmonics voltage can be further calculated from the sum harmonics current at substations as long as the impedance of the transformer at substation is known. The dielectric losses of the capacitors banks at substation can also be found from the voltage harmonics.

Here

$$
x_{S}=\left[\begin{array}{ccc}
x_{S 11} & \ldots & x_{S 1 N} \\
x_{S 21} & \ldots & x_{S 2 N} \\
\ldots & \ldots & \ldots \\
x_{S M 1} & \ldots & x_{S M N}
\end{array}\right] \text { is the matrix of the }
$$

setting out time of each train at each train station, the column of which is the set out instants of $\mathrm{M}$ trains for $j$ th station, while the row is the departure instants for ith train at $\mathrm{N}$ stations.

$$
x_{A}=\left[\begin{array}{ccc}
x_{A 11} & \ldots & x_{A 1 N} \\
x_{A 21} & \ldots & x_{A 2 N} \\
\ldots & \ldots & \ldots \\
x_{A M 1} & \ldots & x_{A M N}
\end{array}\right] \text { is the motoring time of }
$$

each train for each inter-station running.

$$
x_{D}=\left[\begin{array}{ccc}
x_{D 11} & \ldots & x_{D 1 N} \\
x_{D 21} & \ldots & x_{D 2 N} \\
\ldots & \ldots & \ldots \\
x_{D M 1} & \ldots & x_{D M N}
\end{array}\right] \text { is the braking time of }
$$

each train.

$$
x=\left[\begin{array}{lll}
x_{s i j} & x_{a i j} & x_{d i j}
\end{array}\right] \text { is the variable vector that }
$$
determine a special profile of $i$ th train from $j$ th station.

Since the distance between the trains and the traction substations is very short for urban railway system, the parasitic parameters and line impedance of the electrical lines of the traction system can be ignored for the dominant harmonics components. The harmonics current at substation is a vectorial sum of harmonics currents of individual trains,

$$
\vec{I}_{h}=\overrightarrow{I_{h 1}}+\overrightarrow{I_{h 2}}+\ldots+\overrightarrow{I_{h J}}, h=1,3,5, \ldots, 19
$$

where $J$ is the number of trains running within the network powered by the substation concerned.

The Total Harmonic Current Distortion at substations before filter banks is a ratio of harmonics current and fundamental current, which is defined as:

$$
T H D=\frac{I_{H}}{I_{1}}
$$

where the harmonics current is:

$$
I_{H}=\sqrt{\sum_{h=2}^{H} I_{h}^{2}}
$$

The distance between each two successive stations are:

$$
d=\left[\begin{array}{llll}
d_{1} & d_{2} & \ldots & d_{N}
\end{array}\right]
$$

The variables are subject to the following constraints.

Sequence constraints: The displacement of the trains in the same segment of the track should follow the sequence constraints, that is, the train setting out later must run after the train ahead for a minimum safety distance.

Displacement constraints: The displacement of a train with a prescribed speed profile should be accurate enough to meet the equation:

$$
d=\frac{1}{2} a \cdot x_{A}^{2}+a \cdot x_{A} \cdot x_{C}+\frac{1}{2} a^{\prime} \cdot x_{D}^{2}
$$

or the error should be less than a tolerable value.

In order to combine the constraints into the optimization problem, penalty function is introduced. The fitness function will be made up of two parts, the objective function and the penalty function,

$$
\text { fitness }=o b j\left(x_{S}, x_{A}\right)+w \cdot p\left(x_{S}, x_{D}\right)
$$

where $w$ is a penalty weight factor.

\section{GENETIC ALGORITHM}

$\left[x_{S}, x_{A}, x_{D}\right]$ consists of a solution space and the search of the potential solutions can be regarded as a conventional optimization problem. As is known, for small spaces, classical exhaustive methods usually suffice for such problem. However, if the solution space is very large, exhaustive method become impossible because the computation time become extremely long. Special artificial intelligence technique such as evolutionary algorithms can be employed. Genetic Algorithm is one of those stochastic algorithms whose search methods simulate some natural phenomena: genetic inheritance and Darwinian strife for survival.

The structure of a typical genetic algorithm can be shown in Fig 3. The main function blocks include initialization, evaluation, termination, selection, crossover and mutation. Normally GA includes four main steps:

1) Parameter set coding

When GA is applied to an optimization problem, it is necessary to represent the solutions in a string form which is 
regarded as chromosome or individual. GA operates on the segments of the strings to find the optimal solution. Generally, binary encoding is used. The choice of the length of the code is a compromise of the computation expense and the precision of the solution. For example: for a variable $x \in\left(x_{\min }, x_{\max }\right)$ and use $\mathrm{N}$ bits to represent this variable, then candidate solution of $x$ are linearly mapped into binary values, with the minimum and maximum ones as:

$$
\begin{aligned}
& x_{\min } \rightarrow \underbrace{000 \ldots 000}_{N} \\
& x_{\max } \rightarrow \underbrace{111 \ldots 111}_{N}
\end{aligned}
$$

To combine all the variables together to form a chromosome, it will be like this:

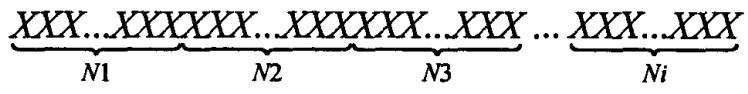

where $X \subseteq\{0,1\}$ and $N_{i}$ is the bits of binary code used for the $i$ th variable.

The value of $\mathrm{x}$ can be decoded from the binary number after optimization is finished. This is also called decoding. Obviously, the resolution of the solution for th variable is:

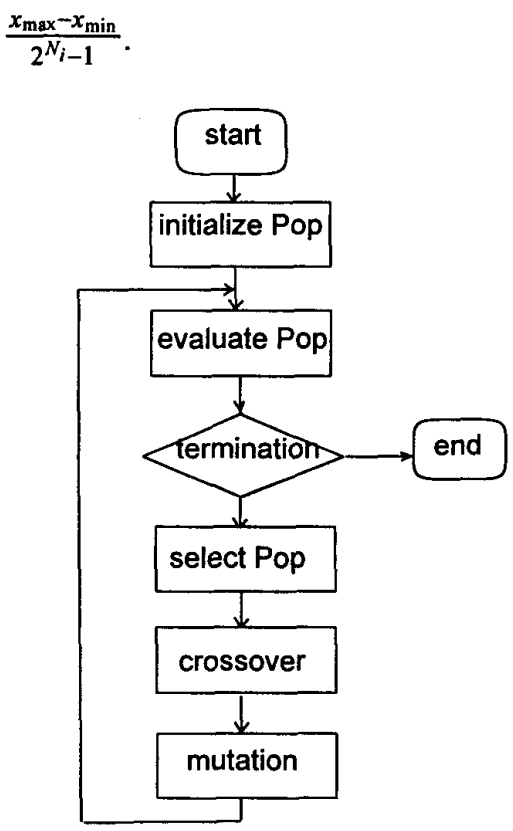

Fig 3. Typical flowchart of GA

2) Initialization of the population

To generate a first population. Usually, uniform distribution is used to select a candidate solution from the pool of the binary numbers. Each individual represents a potential solution to the problem at hand. The size of the population should not be too small, and should be chosen with regard to the number of variables.

\section{3) Objective function evaluation}

Each member of the population is evaluated with a fitness function. Normally, for an electrical system, if direct relation between the fitness and the variables is unavailable, the fitness can be obtained by simulation based on mathematical model of the system.

4) Population reproduction, including selecting, crossover and mutation.

The evolutionary algorithm maintains a population of individuals for every iteration. In the above step, each solution is evaluated to give some measure of its fitness. Then a new population is formed by selecting the more fit individuals. Some members of the new population undergo transformations by meaning of genetic operators to form new solutions.

There are three genetic operators, include crossover, mutation and selection. Crossover of two random pairs of individuals with certain possibility will exchange the genes of individuals, thus produce new individuals, while mutation will produce new genes.

Crossover operators are important for GA. For binary coding, firstly select two individuals from mating pools, and determine if crossover is necessary according to crossover probability. And then set the crossover point, exchange the genes of the individuals before or after this point accordingly. A one-point crossover is demonstrated below:

$$
\begin{aligned}
& A \text { 1001: } 111 \stackrel{\text { crossover }}{\longrightarrow} \text { 1001: } 000 A^{\prime} \\
& B \text { 0011: } 000 \stackrel{\longrightarrow}{\longrightarrow} \text { 0011: } 111 B^{\prime}
\end{aligned}
$$

The crossover is taken place at the crossover point between locus 4 and 5 of individual $A$ and $B$. When crossover, the two individuals exchange the codes beyond this point. Thus two new individuals $A^{\prime}$ and $B^{\prime}$ are generated. In the above, the crossover point is randomly set and crossover can be performed more than one time for two individuals. The crossover is one of the most frequent operations in GA.

The mutation operator ensures the diversity of population and gives GA the ability of local search ability. The probability of mutation is always very low and change of the fitness by mutation is always limited compared with crossover. However, new genes can be introduced by mutation operations. One of the mutation operator for binary codes is to invert the genes of some locus of the chromosome according to some mutation probability. For instance:

$$
A 1011011 \stackrel{\text { mutation }}{\rightarrow} 1110011 A^{\prime}
$$


In the above, the genes at locus 2 and 4 of chromosome $A$ are inverted. Thus a new chromosome $A^{\prime}$ is generated. The locus of the genes for mutation are randomly chosen.

Tournament method, roulette method, elitism method, rank-based method are the commonly used selection mechanism to select the more fit individuals from the pool of individuals. The roulette method, also called fitness proportional method or Monte Carlo method, determine the probability of individuals to be chosen in proportion of their fitness. For an individual $i$ with fitness $f_{i}$, the probability of $i$ to be $\operatorname{chosen} p_{s i}$ is:

$$
p_{s i}=\frac{f_{i}}{\sum_{j=1}^{M} f_{j}}
$$

Obviously, the larger the fitness of the individual, the higher the probability the individual to be chosen. Thus, the more fit chromosome has more chance to survive and more priority to pass its genes to the offspring.

The selected individuals form a new generation with the same size of population of the one proceeded. This simulates the evolution of the nature genetics which the chromosomes passes continuously from generation to generation while the environment selects the best ones to pass on and mutation ensures that new individuals will be introduced to the group to ensure the varsity of the population. After some number of generations, the algorithm converges to the optimal solution. It is hoped that the best individual represents a near optimum solution.

\section{EXAMPLE AND OPTIMIZATION RESULTS}

For the urban railway traction system studied, the traction drives draw power from $132 / 25 \mathrm{KV}, 50 \mathrm{~Hz}$ transformer at two substations. For one substation, there are 7 railway stations that get power from it. The distance of the successive stations are shown in Table I. The timetable of the trains for a portion of a day for the fall season is given in Table II.

TABLE I

THE DISTANCE BETWEEN STATIONS

\begin{tabular}{|c|c|c|c|c|c|c|}
\hline station & $1-2$ & $2-3$ & $3-4$ & $4-5$ & $5-6$ & $6-7$ \\
\hline distance $(\mathrm{Km})$ & 2.45 & 1.85 & 4.35 & 1.4 & 1.95 & 2.38 \\
\hline
\end{tabular}

The minimum and maximum values of the motoring time for each such inter-station run are obtained using practical monitored data. Samples for motoring time and running time are recorded and the minimum and maximum values can be defined according to the $5 \%$ and $95 \%$ points of the cumulative probability density function of the motoring times. The probability density functions of the motoring times and running time for the second inter-stations are shown in Fig. 4.
TABLE II

TIMETABLE OF THE TRAINS FOR 7 STATIONS FOR A CERTAIN PERIOD OF TIME OF A DAY (unit: hour)

\begin{tabular}{|c|c|c|c|c|c|c|c|c|}
\hline $\begin{array}{c}\text { stat. } \\
\text { no. }\end{array}$ & $\begin{array}{c}\text { train } \\
\# 1\end{array}$ & $\begin{array}{c}\text { train } \\
\# 2\end{array}$ & $\begin{array}{c}\text { train } \\
\# 3\end{array}$ & $\begin{array}{c}\text { train } \\
\# 4\end{array}$ & $\begin{array}{c}\text { train } \\
\# 5\end{array}$ & $\begin{array}{c}\text { train } \\
\# 6\end{array}$ & $\begin{array}{c}\text { train } \\
\# 7\end{array}$ & $\begin{array}{c}\text { train } \\
\# 8\end{array}$ \\
\hline 1 & 0.39 & 0.44 & 0.5 & 0.56 & 1.02 & 1.09 & 1.14 & 1.2 \\
\hline 2 & 0.42 & 0.47 & 0.53 & 0.59 & 1.06 & 1.13 & 1.18 & 1.24 \\
\hline 3 & 0.45 & 0.5 & 0.56 & 1.02 & 1.09 & 1.15 & 1.2 & 1.26 \\
\hline 4 & 0.47 & 0.52 & 0.57 & 1.04 & 1.11 & 1.17 & 1.22 & 1.28 \\
\hline 5 & 0.51 & 0.56 & 1.02 & 1.09 & 1.15 & 1.22 & 1.26 & 1.33 \\
\hline 6 & 0.54 & 0.59 & 1.04 & 1.11 & 1.18 & 1.24 & 1.29 & 1.35 \\
\hline 7 & 0.57 & 1.03 & 1.09 & 1.15 & 1.22 & 1.28 & 1.33 & 1.39 \\
\hline
\end{tabular}

a)

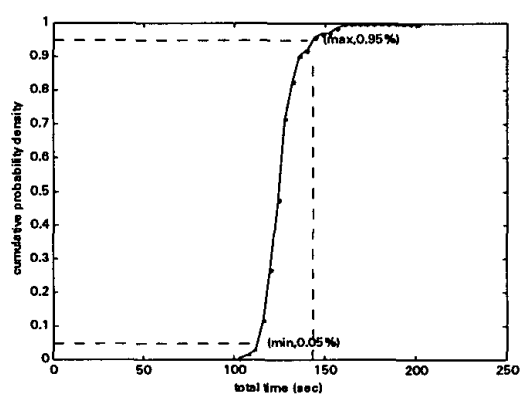

b)

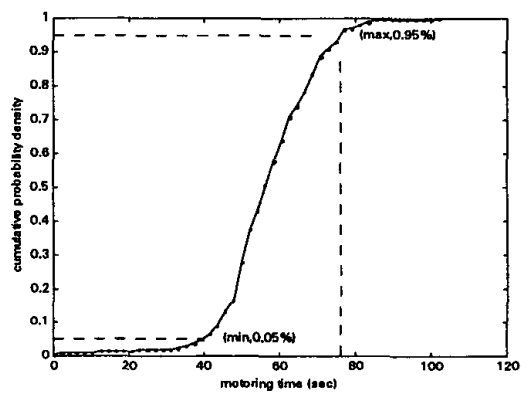

c)

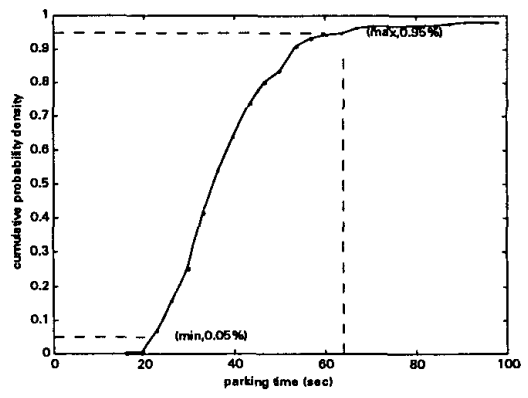

Fig. 4. The probability density function of motoring time (b), total time (a) and parking time (c) for \#2 inter-station runs.

Therefore, the maximum and minimum motoring time for trains can be obtained in Table III for each inter-stations. In the same way, the limit of running time can also be determined. 
TABLE III

THE MEAN AND VARIANCE OF THE MOTORING TIME AND RUNNING TIME FOR INTER-STATION RUNS

\begin{tabular}{|c|c|c|}
\hline station & $\begin{array}{l}\text { motoring time } \\
\text { (unit: sec) }\end{array}$ & $\begin{array}{l}\text { running time } \\
\text { (unit: sec) }\end{array}$ \\
\hline $1-2$ & {$\left[\begin{array}{ll}50 & 140\end{array}\right]$} & {$\left[\begin{array}{ll}100 & 250\end{array}\right]$} \\
\hline $2-3$ & {$\left[\begin{array}{ll}30 & 90\end{array}\right]$} & {$[100$} \\
\hline $3-4$ & {$\left[\begin{array}{ll}60 & 180\end{array}\right]$} & {$\left[\begin{array}{ll}200 & 300\end{array}\right]$} \\
\hline $4-5$ & {$\left[\begin{array}{ll}25 & 80\end{array}\right]$} & 150] \\
\hline $5-6$ & {$\left[\begin{array}{ll}40 & 95\end{array}\right]$} & {$\left[\begin{array}{ll}110 & 160\end{array}\right]$} \\
\hline $6-7$ & {$\left[\begin{array}{ll}40 & 120\end{array}\right]$} & {$[150 \quad 210]$} \\
\hline
\end{tabular}

Genetic Algorithm can now be employed to find the optimal solution such that the total harmonics current or average harmonic current at substation is minimum, with the variables fall in the limit defined above.

For one example to find an optimal solution so that the average harmonics current at substations be minimized. The design variables include starting time and motoring time of 8 trains. Altogether there are 98 variables, which is almost impossible for exhaustive method to find the optimal solution. The probability for mutation is 0.06 and the number of crossover is 80 . After 180 generations, the fitness converge to a final solution as shown in Fig. 5 .
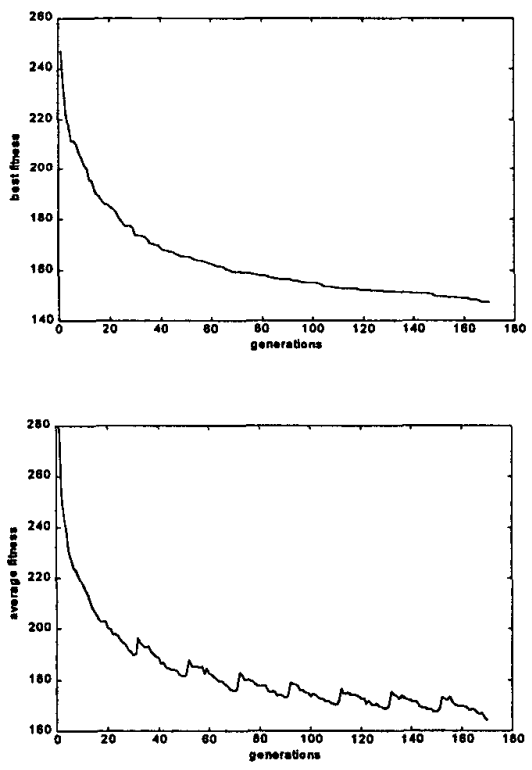

Fig. 5. The trace of the best fitness and average versus generations

Fig. 5 -a shows the trace of the fitness of the best solution for each generation while Fig. 5-b shows the trace of the average fitness of each generation. For this traction problem, the highest frequency considered is 13th. And the minimization problem is transformed to its equivalent maximization problem. The minus of the fitness function is the sum of harmonic current (unit: p. u.) of 3rd, 5th, 7th, 9th,
11 th and 13th combined with penalty function at all sampling points. The harmonic current can be reduced to $12 \%-20 \%$ of that of the original schedule, depends on the starting state of the optimization. Compared with the probabilistic value of the same system, the average harmonics current of the optimized solution is below the mean value of that of the system with traditional schedule, where the departure time is confined to the timetable of the traction railway company and the motoring times of each train are randomly distributed as described above.

For another example to optimize the overall current THD at substation, the minimum THD of the first generation is 0.46 , which implies not much harmonic cancellation. The mean value of the Total Harmonic Distortion is 0.32 but after 120 generations, the minimum THD is 0.24 , which means that the optimized schedule leads to $30 \%$ of reduction of total harmonic distortion of current.

\section{CONCLUSION}

To summarize, reducing the harmonic pollution by scheduling of the trains is a possible approach while not requiring extra equipment investment. This problem is mathematically described. Genetic Algorithm is a promising technique to solve these kinds of complicated optimization problems. The GA optimizes the starting time and motoring time of each train from each station. Such method of harmonics reduction can bring about considerable saving in filtering equipment.

\section{ACKNOWLEDGMENT}

The authors would like to acknowledge the Kowloon Canton Railway Corporation for their help and advice throughout this project.

\section{REFERENCES}

1] T. K. Ho, J. P. Norton, C. J. Goodman, Optimal traffic control at railway junctions, IEE Proc. Electrical Power Application, Vol.144, No. 2, March 1997, pp 140-148.

2] C. S. Chang, F. Wang, K. S. Lock, Harmonic worst-case identification and filter optimal design of MRT systems using genetic algorithms, IEE Proc. Electrical Power Application, Vol. 144, No.5, Sept. 1997, pp 372-380. 3] Ye Z., Pong M. H., Lo Edward, Yuen K. H., Harmonic evaluation of traction system by Monte Carlo simulation, to appear in IEEE PESC 99 conference.

4] Holland J. H., Adaptation in Natural and Artificial Systems. The University of Michigan Press, 1975.

5] Booker, Goldberg, Holland, Classifier Systems and Genetic Algorithms, Artificial Intelligence, 1989, pp 235-282. 